\title{
Describing the Clinical and Laboratory Features and HLA-B Pattern of Adult-Onset Idiopathic Autoimmune Uveitis at a Tertiary Hospital in South India: A Cross-Sectional Study
}

\author{
Jyothi Visalakshy, ${ }^{1}$ Sandeep Surendran ${ }^{1},{ }^{1}$ Salil Ganu $\left(\mathbb{D},{ }^{1}\right.$ Kannisha Shah, ${ }^{2}$ C. B. Mithun, ${ }^{1}$ \\ Vishal Marwaha, ${ }^{1}$ Lalitha Biswas, ${ }^{3}$ Niveditha Kartha $\left(\mathbb{0},{ }^{4}\right.$ and Gopal Pillai ${ }^{2}$ \\ ${ }^{1}$ Department of Rheumatology, Amrita Institute of Medical Sciences, Amrita Vishwa Vidyapeetham, Kochi, Kerala, India \\ ${ }^{2}$ Department of Ophthalmology, Amrita Institute of Medical Sciences, Amrita Vishwa Vidyapeetham, Kochi, Kerala, India \\ ${ }^{3}$ Department of Molecular Biology, Amrita Institute of Medical Sciences, Amrita Vishwa Vidyapeetham, Kochi, Kerala, India \\ ${ }^{4}$ Department of Biostatistics, Amrita Institute of Medical Sciences, Amrita Vishwa Vidyapeetham, Kochi, Kerala, India
}

Correspondence should be addressed to Salil Ganu; gsashokrao@aims.amrita.edu

Received 28 September 2021; Accepted 18 January 2022; Published 8 February 2022

Academic Editor: Ahmad Mansour

Copyright (c) 2022 Jyothi Visalakshy et al. This is an open access article distributed under the Creative Commons Attribution License, which permits unrestricted use, distribution, and reproduction in any medium, provided the original work is properly cited.

\begin{abstract}
Introduction. There is a scarcity of information available on clinical and laboratory features of adult-onset idiopathic autoimmune uveitis. Therefore, we conducted a single centre descriptive cross-sectional study. Patients and Methods. A chart review of all patients with idiopathic autoimmune uveitis with onset after 18 years of age who were referred to the rheumatology department between January 2017 and December 2018 was performed. Their clinical features, demographic features, and HLA-B genotypes were documented and described. Results. Out of 210 patients referred to rheumatology, 66 were found to have uveitis, and 16 of these had an adult-onset idiopathic autoimmune uveitis. Apart from a slight female preponderance (62.5\%), our patients were characterized by a high proportion of panuveitis (4 out of 16, i.e., 25\%). There was an increased frequency of occurrence of synechiae ( 5 out of 16, i.e., $31.3 \%$ ), retinal vasculitis ( 4 out of 16, i.e., $25 \%$ ), optic disc edema ( 3 out of 16, i.e., 18.8\%), and cystoid macular edema (seen in 2 patients, i.e., 12.5\%). These features correlated with the anatomical subtypes. Retinal vasculitis and optic disc edema present in three fourth of all panuveitis cases were the most prominent features. The odds of finding HLA-B*35 in retinal vasculitis were 33 times higher than odds of finding it in idiopathic autoimmune uveitis patients not having retinal vasculitis (OR 33; 95\% CI 1.6-698). Conclusion. Idiopathic autoimmune uveitis in our patients is characterized by a high frequency of panuveitis and retinal vasculitis, and complications with a probable association between HLA-B $* 35$ and retinal vasculitis.
\end{abstract}

\section{Introduction}

Uveitis is an important cause of ocular morbidity throughout the world leading to $5-10 \%$ of all causing visual impairment [1]. About one third of all cases of uveitis result in a significant visual loss [2]. Nearly 17-52 new cases of uveitis per 100,000 population occur in the world with a prevalence of 38-714 cases per 100,000 population [3]. India alone has a prevalence of 730 uveitis cases per 100,000 population, and a tertiary care center in India reported an incidence of $1.5 \%[4,5]$. There is a regional difference documented in different parts of India. The common complications of uveitis include cataract, glaucoma, band keratopathy, cystoid macular edema, optic disc edema, vitreal hemorrhages, epiretinal membrane, and retinal neovascularization [6-10]. Retinal vasculitis is seen accompanying a severe form of posterior uveal inflammation. Despite the burden of this problem, the classification of uveitis has always been an ever-evolving process due to the discovery of new diagnostic modalities. There are various ways in which uveitis has been traditionally classified. These include anatomical classification, classification based on chronicity, and etiological classification. Most recent etiological classification schemes classify uveitis into infectious and noninfectious varieties. The noninfectious uveitis is further classified into 
TABLE 1: Classification criteria used for systemic autoimmune diseases associated with uveitis.

\begin{tabular}{lc}
\hline Disease & Classification or diagnostic criteria \\
\hline Spondyloarthropathy & ASAS \\
Psoriatic Arthritis & CASPER \\
Systemic Lupus Erythematosus & SLICC2012 \\
Behcet's disease & ICBD2006 \\
Rheumatoid arthritis & ACR2010 \\
ANCA-associated vasculitis & ACR1990 \\
Sarcoidosis & Clinical diagnosis confirmed by histological evidence \\
\hline
\end{tabular}

malignancy-related and autoimmune varieties. The subsequent classification of autoimmune uveitis is performed on the basis of its association with systemic autoimmune disease as a systemic disease-associated autoimmune uveitis and idiopathic autoimmune uveitis [11]. An Indian study from 1996 reports that the etiology of uveitis is established in $59.31 \%$ of the cases [4]. The percentage of which is likely to be increasing in subsequent decades. Although there has been ample literature describing the burden of noninfectious uveitis and its complications, the literature addressing the same in autoimmune uveitis as a group has been scarce. The lack of availability of the literature pertaining to prevalence, natural history, complications, and sequelae is particularly remarkable for idiopathic-autoimmune variety of autoimmune uveitis. In our clinical experience, we found that the idiopathic autoimmune uveitis has a fairly distinct clinicolaboratory phenotype with a high frequency of various complications and the associated retinal vasculitis. Therefore, we have tried to describe our experience with adult-onset idiopathic autoimmune uveitis and its complications with a special reference to retinal vasculitis.

1.1. Objective. The objective of this study is to describe the clinical and laboratory features of an idiopathic autoimmune uveitis.

\section{Patients and Methods}

2.1. Study Design and Patient Selection. A screening of the electronic medical hospital records was done for all patients who consulted the rheumatology department of a tertiary care hospital in south India between January 2017 and December 2018. Among the screened charts, the patients diagnosed to have uveitis by an expert ophthalmologist were selected. Uveitis was considered to be idiopathic autoimmune uveitis if the following criteria were fulfilled: (1) all known causes of infectious uveitis had been ruled out; (2) the systemic autoimmune diseases such as sarcoidosis, Behcet's disease, spondyloarthropathy, or connective tissue disorder syndromes (SLE, RA, and Sjogren's syndrome) were excluded by a rheumatologist on clinical grounds as well as with respective classification criteria (enlisted in, Table 1); (3) a favorable expert opinion from an ophthalmologist for the diagnosis of idiopathic autoimmune uveitis was taken; and (4) malignancies were ruled out whenever suspected patients were selected if the onset of their first uveitis symptom happened after 18 years of age. All patients who were residents of the state of Kerala were included because it is a tourist center. All patients with idiopathic autoimmune uveitis were treated in a multidisciplinary setting as a part of the institutional standard of care, and investigations for determining the cause of the uveitis were carried out in all patients in a tailored approach $[12,13]$. All the idiopathic autoimmune uveitis patients who were diagnosed in such a manner were eligible for enrollment after confirming verbal (telephonic) informed consent. Patients were enrolled in the study if adequate details of their clinical features and treatment were available. Recruited patients were further classified using the Standardization of Uveitis Nomenclature (SUN) Working Group of anatomical classes of uveitis [14]. The characteristic features and treatments received by idiopathic autoimmune uveitis patients in general and in their anatomical subclasses were described.

2.2. Clinical Details and Outcome Measures. Basic demographic details (age and gender) were noted. Duration of disease and past treatment history including the response to previous treatment were documented. Uveitis was classified according to anatomical localization as anterior (iritis, iridocyclitis, and anterior cyclitis), intermediate (pars planitis), posterior (focal, multifocal, or diffuse choroiditis, chorioretinitis, retinochoroiditis, and neuroretinitis), or panuveitis (inflammation of the anterior chamber, vitreous, and retina or choroid). In addition, information about acute, chronic, or recurrent nature and laterality (unilateral Vs bilateral) of uveitis was also collected. Extraocular features were also documented. Examination findings such as the presence of synechiae, vasculitis, retinal hemorrhages, hypopyon, cataract, epiretinal membranes, and features suggestive of glaucoma were also noted. The baseline CRP (mg/L) and ESR $(\mathrm{mm} / \mathrm{Hr}$.) levels and the nature of treatment received (topical/local, oral, or parenteral steroids, DMARD initiation, etc.,) were documented. Change in visual acuity was documented whenever data were available.

2.3. HLA-B Genotype Analysis Procedure. Genomic DNA is extracted from the peripheral blood samples using the salting-out method. The DNA obtained is quantified using the spectrophotometer (biophotometer, Germany) and adjusted to a concentration of $30 \mathrm{ng} / \mu \mathrm{l}$. HLA-B tissue typing is performed using the HLA-B sequence-specific primerbased low-resolution kit (BAG Healthcare, Germany) according to the manufacturer's protocol. The PCR products are electrophoresed in a 3\% agarose gel. HLA alleles are assigned based on the band pattern using the HistoMatch software (BAG Healthcare, Germany). 


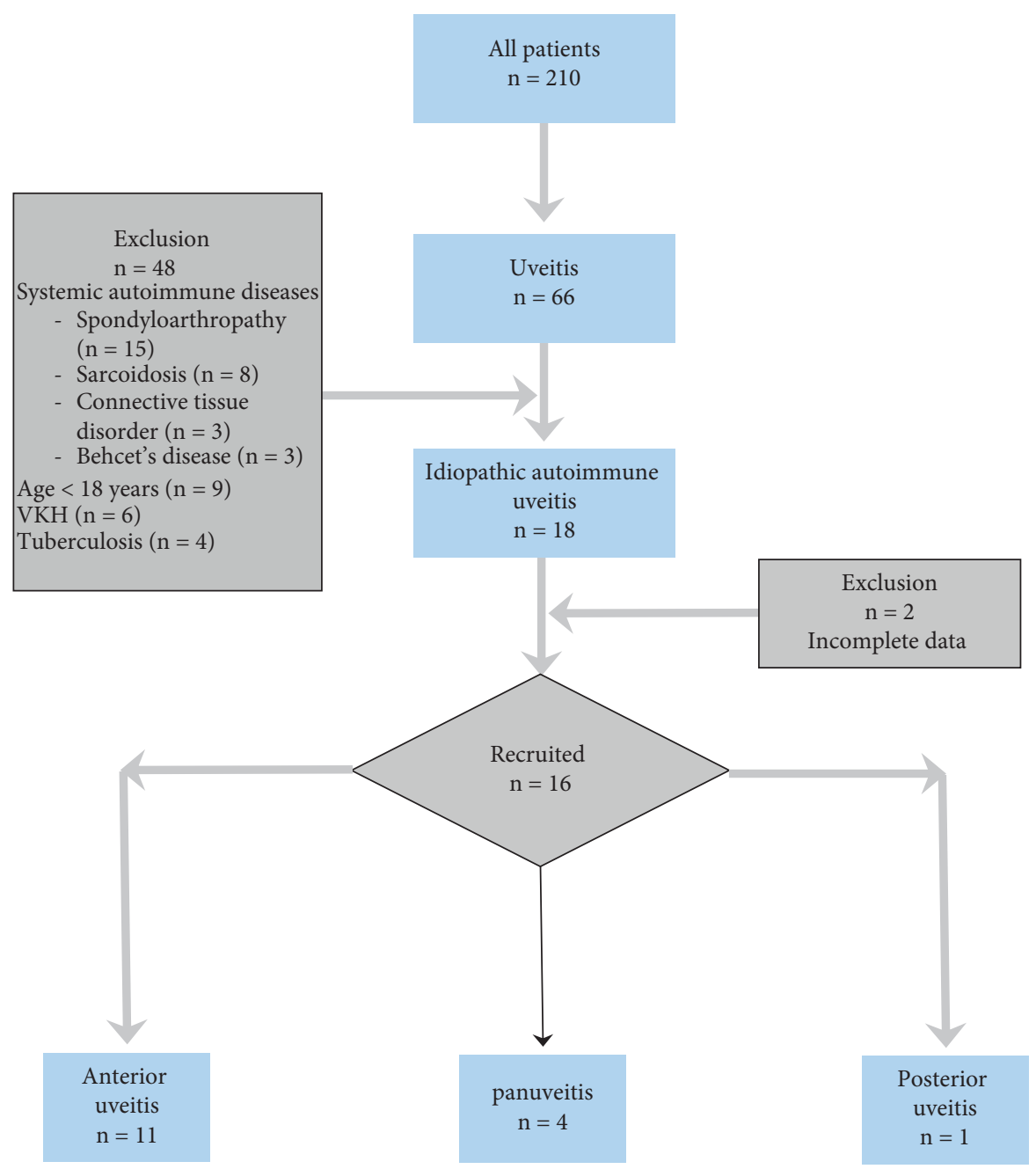

Figure 1: Patient flow in the study.

2.4. Statistical Methods. The analysis was carried out using SPSS25 software. Descriptive statistics were summarized using means and percentages.

\section{Results}

Charts of a total of 210 patients who were referred to the rheumatology department for the evaluation of autoimmune uveitis were screened. Total 66 patients were found to have a diagnosis of uveitis, out of which 48 patients were excluded, and 18 patients were found to have a diagnosis of idiopathic autoimmune uveitis. Two patients who did not have a complete clinical record were excluded. A total of 16 patients with a diagnosis of idiopathic uveitis were selected. All patients included in this study had recognized themselves as ethnical south Indians. Figure 1 summarizes the patient flow in the study. The baseline demographic characteristics and disease profile of all patients are described in Table 2. The median age of autoimmune idiopathic uveitis patients was found to be 41 (37-58.2) years. There was a female preponderance among idiopathic autoimmune uveitis cases, and the median disease duration was $24(6.3-36)$ months. Eleven patients had anterior uveitis, 4 had panuveitis, and 1patient had posterior uveitis. At the time of their first visit, the previous treatment record was available for 13 patients, out of which 3 patients were treatment naïve, 8 had received some form of treatment including 4 patients each being treatment refractory and treatment responsive. Refractoriness was defined as no inactivity or reactivation of uveitis at doses of topical steroid of $>3$ drops per day given for at least 12 weeks. [15] The diagnosis was confirmed in all patients as mentioned in the methodology section. Three patients including two having anterior uveitis and one having panuveitis were found to have a tuberculin sensitivity test of more than $5 \mathrm{~cm}$ but less than $10 \mathrm{~cm}$. These did not have characteristic pulmonary or extrapulmonary features of TB. These also did not have specific ocular features. As these patients had a history of BCG vaccination in childhood and tested negative on interferon gamma release assay, they were considered to have inadequate evidence to be classified as having an ocular $\mathrm{TB}$ although tissue despite having no tissue-culture testing. Consent for the same was refused by the patient. One patient among these did receive empirical antitubercular treatment for latent treatment in anticipation of future need for a TNF-alfa inhibitor. 
TABLE 2: Baseline characteristics.

\begin{tabular}{|c|c|c|c|c|}
\hline Features & $\begin{array}{l}\text { Idiopathic autoimmune uveitis } \\
\qquad(N=16)\end{array}$ & $\begin{array}{l}\text { Anterior uveitis } \\
\quad(N=11)\end{array}$ & $\begin{array}{l}\text { Panuveitis } \\
\quad(N=4)\end{array}$ & $\begin{array}{l}\text { Posterior uveitis } \\
\quad(N=1)\end{array}$ \\
\hline Age in years, median (IQR) & $41(37-58.2)$ & $41(40-60)$ & $41(23.8-65)$ & 37 \\
\hline Female, $n(\%)$ & $10(62.5)$ & $6(54.5)$ & $4(100)$ & - \\
\hline $\begin{array}{l}\text { Uveitis duration in month, median } \\
\text { (IQR) }\end{array}$ & $24(6.3-36)$ & $12(6-36)$ & $30(11.3-126)$ & 36 \\
\hline \multicolumn{5}{|l|}{ Past treatment history } \\
\hline Treatment refractory, $n$ ' $(n)$ & $4(13)$ & $2(8)$ & $2(4)$ & 1 \\
\hline Treatment naïve, $n ’(n)$ & $5(13)$ & $3(8)$ & $1(4)$ & - \\
\hline Treatment responsive, $n^{\prime}(n)$ & $4(13)$ & $3(8)$ & $1(4)$ & - \\
\hline \multicolumn{5}{|l|}{ Tuberculin sensitivity test, n' (n) } \\
\hline $\begin{array}{l}\text { Interferon gamma release assay, } n \text { ' } \\
(n)\end{array}$ & $3(12)$ & $2(7)$ & $1(3)$ & $0(1)$ \\
\hline CXR PA view for TB, $n^{\prime}(n)$ & $0(7)$ & $0(3)$ & $0(1)$ & $0(1)$ \\
\hline Sacroiliac MRI, $n^{\prime}(n)$ & $0(12)$ & $0(9)$ & $0(2)$ & $0(1)$ \\
\hline ANA (IFA), $n^{\prime}(n)$ & $0(6)$ & $0(5)$ & - & $0(1)$ \\
\hline AntiPR3/MPO, n' $(n)$ & $2(5)$ & $1(3)$ & $1(1)$ & - \\
\hline $\mathrm{ACE}, n^{\prime}(n)$ & $0(5)$ & $0(3)$ & $0(1)$ & $0(1)$ \\
\hline CRP, median (IQR) & $0(12)$ & $0(7)$ & $0(4)$ & $0(1)$ \\
\hline ESR, median (IQR) & $5.1(2-8)$ & $5.1(2.4-8)$ & $7.4(2-32)$ & 1.1 \\
\hline Topical treatment & $16(10-40)$ & $18(10-40)$ & $16(15-57)$ & 6 \\
\hline Topical steroid, $n(\%)$ & $15(93.8)$ & $11(100)$ & $4(100)$ & 一 \\
\hline $\begin{array}{l}\text { Topical cycloplegics/mydriatics, } n \\
(\%)\end{array}$ & $12(75)$ & $9(75)$ & $3(75)$ & - \\
\hline \multicolumn{5}{|l|}{ Regional therapy } \\
\hline Intraocular steroid, $n(\%)$ & $1(6.3)$ & - & $1(25)$ & - \\
\hline Systemic steroids $n(\%)$ & $9(56.3)$ & $4(36.4)$ & $3(75)$ & - \\
\hline Intravenous steroid, $n(\%)$ & $4(25)$ & $2(18.2)$ & $1(25)$ & - \\
\hline Oral steroid, $n(\%)$ & $8(50)$ & $3(27.3)$ & $3(75)$ & 11 \\
\hline \multicolumn{5}{|l|}{ Additional immunosuppression } \\
\hline Steroid sparing, $n(\%)$ & $7(43.8)$ & $5(45.5)$ & $2(50)$ & 1 \\
\hline Mycophenolate mofetil, $n(\%)$ & $2(12.5)$ & - & $2(50)$ & 1 \\
\hline Methotrexate, $n(\%)$ & $4(25)$ & $3(27.3)$ & $1(25)$ & - \\
\hline Sulfasalazine, $n(\%)$ & $3(18.8)$ & $3(27.3)$ & - & - \\
\hline Other therapy, $n(\%)$ & $1(6.3)$ & - & $1(25)$ & - \\
\hline $2^{\text {nd }}$ DMARD, $n(\%)$ & $3(18.8)$ & $2(18.2)$ & $1(25)$ & - \\
\hline
\end{tabular}

$n^{\prime}=$ frequency of positive results, $n=$ total number of patients with a variable, $N=$ total number of patients in the group.

Among all idiopathic autoimmune uveitis patients, at the time of patient recruitment in the study, a large majority of our patients had received topical steroids and topical cycloplegics. Systemic steroids, including some form of intravenous steroid in selected patients, were used in a total of 9 patients. Almost half of our patients had received at least one conventional synthetic DMARD (cs DMARD), whereas three had required a second cs DMARD. None of our patients had required a biological DMARD (b DMARD).

\subsection{Clinical and Laboratory Features of Idiopathic Autoim-} mune Uveitis. The clinical and laboratory characteristics of patients are described in Table 3. A slightly above half of our patients had unilateral uveitis. Pain and redness of eyes were the most common symptoms, whereas synechiae formation was the most common complication encountered. A sizeable number of patients had blurring of vision. Among the three patients who had a cataract, one was found to have a probable steroid-induced cataract (with a history of long duration of steroid use), and another one had a probable senile (nuclear sclerosing) cataract. Hypopyon, epiretinal membranes, or glaucoma were not found in any of these patients. Almost one third of these patients had retinal vasculitis most of whom also had a cystoid macular edema and/or optic disc edema. Majority of these patients were found to have panuveitis, and one had posterior uveitis. However, none of these patients had vitreous hemorrhages or retinal neovascularization. Visual acuity data for first and last hospital visits were available for only 8 patients. Moreover, many of these patients were initiated on treatment for uveitis long before their first visit. Since their first visit, visual acuity had improved, remained status quo, and worsened in 3 patients each.

A total of nine patients had at least one extraocular feature, and two had more than one extraocular feature. Inflammatory low backache was present in six patients, joint pain was present in four patients, and one patient had heel pain. Joint pain and heel pain are mechanical in nature. None of these patients with inflammatory low backache had sufficient features to be classified as a spondyloarthropathy as per ASAS criteria. Markers of systemic inflammation were 
TABLE 3: Clinical and laboratory features of idiopathic autoimmune uveitis.

\begin{tabular}{|c|c|c|c|c|}
\hline Features & $\begin{array}{l}\text { Idiopathic autoimmune uveitis } \\
\qquad(N=16)\end{array}$ & $\begin{array}{l}\text { Anterior uveitis } \\
\quad(N=11)\end{array}$ & $\begin{array}{l}\text { Panuveitis } \\
(N=4)\end{array}$ & $\begin{array}{c}\text { Posterior uveitis } \\
(N=1)\end{array}$ \\
\hline Unilateral & $9(56.3)$ & $5(45.5)$ & $3(75)$ & 1 \\
\hline \multicolumn{5}{|l|}{ Symptoms } \\
\hline Pain in eye, $n(\%)$ & $12(75)$ & $10(91)$ & $2(50)$ & - \\
\hline Redness of eye, $n(\%)$ & $12(75)$ & $11(100)$ & $1(25)$ & - \\
\hline Epiphora, $n(\%)$ & $4(25)$ & $4(36.4)$ & - & - \\
\hline Photophobia, $n(\%)$ & $7(43.8)$ & $4(63.6)$ & - & -1 \\
\hline Blurring of vision $n(\%)$ & $6(37.5)$ & $1(9.1)$ & $4(100)$ & - \\
\hline Others, $n(\%)$ & $1(6.3)$ & - & $1(25)$ & \\
\hline \multicolumn{5}{|l|}{ Examination findings } \\
\hline Synechiae, $n(\%)$ & $5(31.3)$ & $4(36.4)$ & $1(25)$ & - \\
\hline Cataracts, $n(\%)$ & $3(18.8)$ & $1(9.1)$ & $2(50)$ & - \\
\hline Retinal vasculitis, $n(\%)$ & $4(25)$ & - & $3(75)$ & 1 \\
\hline $\begin{array}{l}\text { Cystoid macular edema, } n \\
(\%)\end{array}$ & $2(12.5)$ & - & $2(50)$ & - \\
\hline Optic disc edema, $n(\%)$ & $3(18.8)$ & - & $3(75)$ & - \\
\hline Optic neuritis, $n(\%)$ & $1(6.3)$ & - & $1(25)$ & - \\
\hline \multicolumn{5}{|l|}{ Visual Acuity } \\
\hline Improved, n'(n) & $3(9)$ & $1(7)$ & $2(2)$ & - \\
\hline Status quo n' $(n)$ & $3(9)$ & $3(7)$ & - & \\
\hline Worsened, $\mathrm{n}^{\prime}(n)$ & $3(9)$ & $3(7)$ & - & \\
\hline Extraocular features, $n(\%)$ & $9(56.25)$ & $8(72.7)$ & $1(25)$ & 1 \\
\hline Inflammatory LBA, $n(\%)$ & $6(37.5)$ & $5(45.5)$ & - & 1 \\
\hline Joint pain, $n(\%)$ & $4(25)$ & $4(36.4)$ & - & - \\
\hline Heel pain $n(\%)$ & $1(6.3)$ & $1(9.1)$ & - & - \\
\hline More than 1 feature & $2(12.5)$ & $2(18.2)$ & - & - \\
\hline \multicolumn{5}{|l|}{ Inflammatory markers } \\
\hline Raised CRP, $n(\%)$ & $6(37.5)$ & $4(36.4)$ & $2(50)$ & 1.1 \\
\hline Raised ESR, $n(\%)$ & $6(37.5)$ & $5(45.5)$ & $1(25)$ & 6 \\
\hline
\end{tabular}

(a) $n^{\prime}=$ frequency of positive results, $n=$ total number of patients with a variable, $N=$ total number of patients in the group. (b) CRP $>6 \mathrm{mg} / \mathrm{L}$. (c) ESR $>22 \mathrm{~mm} /$ Hr.

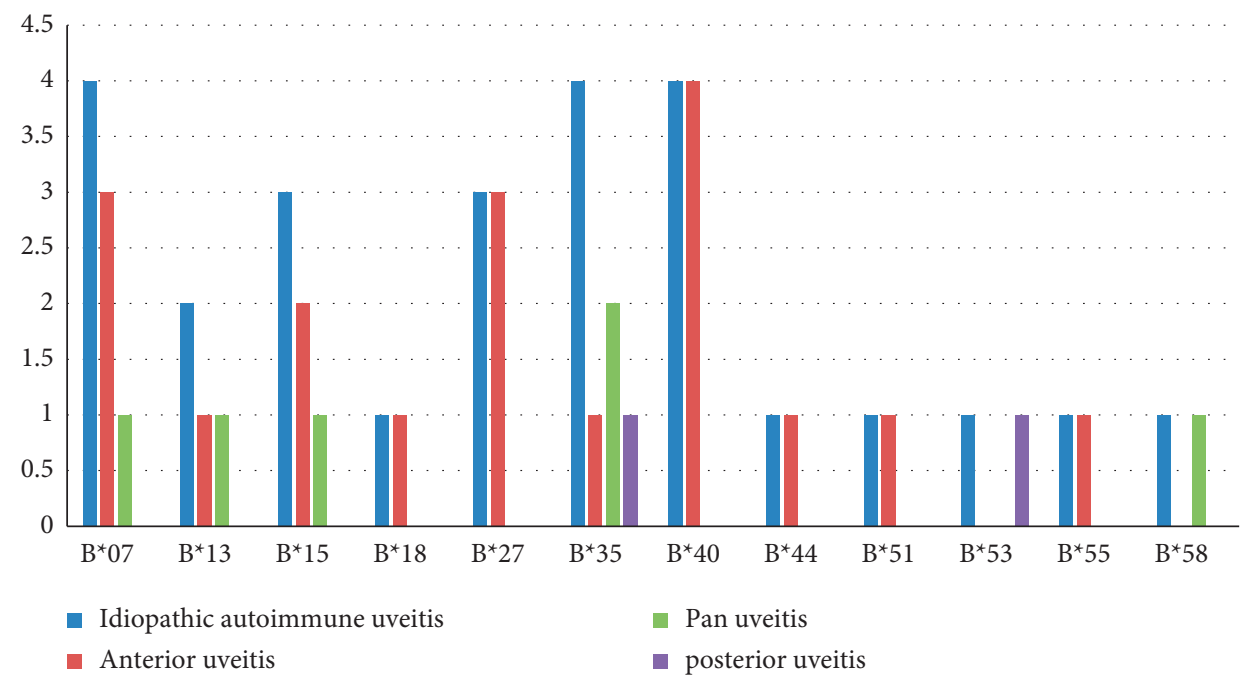

Figure 2: HLA-B typing.

raised in about one third of patients at the time of their first visit. CRP and ESR were raised in 6 patients each. HLA-B type distribution is denoted in Figure 2. HLA-B*07, B*35, and $\mathrm{B} * 40$ were present in 4 patients each. HLA-B $* 35$ was seen in 3 patients who had posterior segment involvement. Two patients were found to have HLA-B*51. However, they did not have adequate clinical features to be classified as Behcet's disease according to ICBD criteria. 
TABLE 4: Clinical and laboratory features of retinal vasculitis.

\begin{tabular}{|c|c|c|}
\hline Features & Retinal vasculitis $(N=4)$ & No retinal vasculitis $\left(N^{\prime}=12\right)$ \\
\hline Age in years, median (IQR) & $33(23.8-49)$ & $45(40-60)$ \\
\hline Female gender, $n(\%)$ & $3(75)$ & $7(58.3)$ \\
\hline Uveitis duration, median (IQR) & $36(27-126)$ & $12(6-34)$ \\
\hline Uveitis laterality unilateral, $n(\%)$ & $4(100)$ & $5(41.7)$ \\
\hline \multicolumn{3}{|l|}{ Uveitis type } \\
\hline Posterior uveitis, $n$ (\%) & $1(25)$ & - \\
\hline Panuveitis, $n(\%)$ & $3(75)$ & $1(8.3)$ \\
\hline \multicolumn{3}{|l|}{ Past treatment history } \\
\hline Treatment refractory, $n^{\prime}(n)$ & $1(4)$ & $3(20)$ \\
\hline Treatment naïve, $n^{\prime}(n)$ & $2(4)$ & $3(10)$ \\
\hline Treatment responsive, $n^{\prime}(n)$ & $1(4)$ & $3(40)$ \\
\hline \multicolumn{3}{|l|}{ Symptoms } \\
\hline Pain, $n(\%)$ & $2(50)$ & $10(83.3)$ \\
\hline Redness, $n(\%)$ & $1(25)$ & $11(91.7)$ \\
\hline Epiphora, $n(\%)$ & - & $4(33.3)$ \\
\hline Photophobia, $n(\%)$ & - & $7(58.3)$ \\
\hline Blurring of vision, $n(\%)$ & $4(100)$ & $2(16.7)$ \\
\hline Black spots, $n(\%)$ & $1(25)$ & - \\
\hline \multicolumn{3}{|l|}{ Examination findings } \\
\hline Synechiae, $n(\%)$ & - & $5(41.7)$ \\
\hline Cataract, $n(\%)$ & $1(25)$ & $2(16.7)$ \\
\hline Cystoid macular oedema, $n(\%)$ & $1(25)$ & $1(6.3)$ \\
\hline Optic disc oedema, $n(\%)$ & $2(50)$ & $1(6.3)$ \\
\hline Vitreous hemorrhages, $n(\%)$ & - & - \\
\hline Optic neuritis, $n(\%)$ & $1(25)$ & - \\
\hline \multicolumn{3}{|l|}{ Extraocular features } \\
\hline Inflammatory low backache & $1(25)$ & $5(41.7)$ \\
\hline Heel pain & $1(25)$ & $1(6.3)$ \\
\hline Joint pain & - & $4(33.3)$ \\
\hline More than 1 feature & - & $2(16.7)$ \\
\hline \multicolumn{3}{|l|}{ Inflammatory markers } \\
\hline CRP, median (IQR) & $1.6(1.1-32)$ & $5.4(2.6-7.9)$ \\
\hline ESR, median (IQR) & $15.5(8.3-46.8)$ & $18(10-40)$ \\
\hline \multicolumn{3}{|l|}{ HLA-B patterns } \\
\hline $\mathrm{B} 07, n$ & 0 & 4 \\
\hline $\mathrm{B} 13, n$ & 1 & 1 \\
\hline $\mathrm{B} 15, n$ & 0 & 3 \\
\hline $\mathrm{B} 18, n$ & 0 & 1 \\
\hline $\mathrm{B} 27, n$ & 0 & 3 \\
\hline $\mathrm{B} 35, n$ & 3 & 1 \\
\hline $\mathrm{B} 40, n$ & 0 & 4 \\
\hline $\mathrm{B} 44, n$ & 0 & 1 \\
\hline $\mathrm{B} 51, n$ & 0 & 2 \\
\hline $\mathrm{B} 52, n$ & 0 & 0 \\
\hline $\mathrm{B} 53, n$ & 1 & 0 \\
\hline B55, $n$ & 0 & 1 \\
\hline B58, $n$ & 1 & 0 \\
\hline
\end{tabular}

$n^{\prime}=$ frequency of positive results, $n=$ total number of patients with a variable, $N=$ total number of patients in the group 3.

3.2. Clinical and Laboratory Characteristics of Retinal Vasculitis. A sizeable number of patients in this study were found to have retinal vasculitis. Therefore, the clinical and laboratory features of retinal vasculitis are described in Table 4 . There were 4 retinal vasculitis patients in this study out of which 3 had panuveitis, and one had posterior uveitis. The clinical features represented the anatomical type of uveitis; therefore, blurring of vision followed by pain and redness of eyes were the most common symptoms noticed. Optic disc edema was the most common complication apart from optic neuritis, cataract, and cystoid macular edema present in one patient each. One patient each had inflammatory low backache and mechanical heel pain. It was also noticed that $\mathrm{HLA}-\mathrm{B} * 35$ was present in 3 patients with retinal vasculitis, indicating that there are 33 times higher odds of having the presence of HLA-B $* 35$ among patients with 
retinal vasculitis as compared to autoimmune idiopathic uveitis patients not having retinal vasculitis (OR 33; 95\% CI 1.6-698).

\section{Discussion}

Indian studies report the prevalence rate for endogenous uveitis as $310 / 100,000$ population with posterior uveitis involvement as a major risk factor for blindness [16]. Indian cohort studies also report etiologies of uveitis, and studies from both north and south India report that idiopathic uveitis accounts for $15-20 \%$ of patients presenting to tertiary ophthalmological clinics $[17,18]$. The literature review shows that a classification scheme similar to ours classifying the uveitis into an idiopathic autoimmune variety has been carried out previously by Prete et al. [11]. In this tertiary center-based retrospective analysis of 104 patients from Italy, a comparative description of idiopathic autoimmune uveitis with systemic disease-associated autoimmune uveitis has been given.

Our study was characterized by the predominance of anterior uveitis (68.8\%) and panuveitis (25\%) as compared to posterior uveitis (6.3\%). This was different from that found in a larger study conducted in the south Indian population having uveitis by Biswas et al. [4]. In this study, they found that anterior uveitis was found in $40 \%$, posterior uveitis in 29\%, intermediate uveitis in $17 \%$, and panuveitis in $14 \%$ of all patients. In the study by Prete et al., anterior uveitis was seen in $42.7 \%$ patients, posterior uveitis in $46.7 \%$, intermediate uveitis in $6.7 \%$, and panuveitis in $10.3 \%$ patients. Another large systematic review by Barisani-Asenbauer et al. also described the prevalence of panuveitis in the range of $2.38 \%$ [19]. This pattern of the higher percentage of anterior uveitis and panuveitis cases was reflected in the frequency of symptoms and complications of uveitis in our study. Consistent with findings in the study conducted by Prete et al., the redness of eyes, pain, and blurring of vision were the predominant complaints in our patients.

In our study, it was found that patients having anterior uveitis had clinical features similar to spondyloarthropathyrelated typical (HLA-B $* 27$ associated) acute anterior uveitis, such as acute presentation with a unilateral or alternating bilateral pattern, recurrent nature, association with HLA B27, and presence of inflammatory low backache $[20,21]$. An odd feature noted was the female preponderance in patients having a typical acute anterior uveitis. We found that there is a mild female preponderance in our study. Another Indian study in patients with uveitis indicates male preponderance worldwide although it is considered to be having an equal distribution [16]. A retrospective study from Holland showed that about $53 \%$ panuveitis, $41 \%$ of intermediate uveitis, and $28 \%$ posterior uveitis had cystoid macular edema [22]. We found that it was present in 12.5\% of all uveitis patients and $75 \%$ of patients having panuveitis. The prevalence of cataract, which is another major complication, is reported to be in $18-35 \%$ of all uveitis cases [23]. This was comparable to our study where $18.8 \%$ patients developed cataract. The prevalence of retinal vasculitis among uveitis cases was found to be $14.9 \%$ in a previous study [24]. This is much lower than in our patients with idiopathic autoimmune uveitis. The higher proportion of retinal vasculitis was probably attributable to the presence of a higher number of patients with a posterior segment involvement. Overall, the percentage of patients who developed various complications of uveitis in our patients was also much higher than that described in the literature [20].

The HLA-B $* 35$ was the most frequent HLA-B serotype present in panuveitis and posterior uveitis patients. HLA$\mathrm{B} * 35$ is one of the biggest HLA-B serotype groups in existence. There are currently 86 polypeptide isoforms and 97 nucleotide variants present in this group [25]. Apart from its well-established association with the accelerated progression of HIV infection to AIDS, HLA-B $* 35$ has been reported to be associated with Juvenile idiopathic arthritis-related uveitis, self-limiting unclassified rheumatism, sacroiliitis in undifferentiated spondyloarthropathy, and oral aphthosis [26-32]. The literature on ocular involvement in HLA-B $* 35$ positivity is scarce and shows its presence in up to $24 \%$ of uveitis patients in a small-sized prospective study [33]. We did not find any previous report of the association of retinal vasculitis with $\mathrm{HLA}-\mathrm{B} * 35$. HLA-B $* 35$ is responsible for endoplasmic reticulum stress response by upregulation of endothelin 1 and downregulation of nitric oxide in endothelial cells of pulmonary vasculature [34]. This causes endothelial cell dysfunction and subsequent pulmonary vasoconstriction. Therefore, whether a similar process of endothelial stress and unfolded protein response associated with $\mathrm{HLA} * \mathrm{~B} 35$ causes inflammation of retinal blood vessels in posterior uveitis remains to be seen. Another mechanism which can be considered plausible is like arthritogenic peptide hypothesis in spondyloarthropathy. Evidence for this comes from the seminal study conducted by Petty et al. on JRA children. In this study, they found that the JRA children with uveitis who had the presence of HLA$\mathrm{B} * 35$ had a higher frequency of antibody to soluble retinal antigen. [27] This might be a result of a process in which HLA-B $* 35$ shows a preferential binding to soluble retinal antigens and their subsequent presentation to lymphocytes causing an abnormal immune response against them like that seen in the case of HLA-B $* 27$ and arthritogenic peptides [35].

There are two main limitations of our study, namely (1) a small number of patients and (2) the possibility of a referral bias. Although this limits the generalizability of our study results, it lays the foundation for generating a hypothesis that adult-onset idiopathic autoimmune uveitis is characterized by a higher percentage of panuveitis and its complications, particularly with retinal vasculitis. It also elucidates the increased odds of the presence of HLA-B $* 35$ among patients with retinal vasculitis. These conclusions can be tested in a prospective cohort study.

\section{Conclusion}

Adult-onset idiopathic autoimmune uveitis in our patients from southern India is characterized by the high frequency of panuveitis, retinal vasculitis, and complications. Also, 
there are higher odds of having the presence of HLA-B $* 35$ in patients having retinal vasculitis.

\section{Data Availability}

Data used in this study are available from the corresponding author upon request.

\section{Ethical Approval}

The study protocol has been approved by Institutional Ethics Committee and performed in accordance with Principles laid down in Helsinki declaration 1964 and its subsequent amendments.

\section{Consent}

A telephonic (verbal) informed consent was obtained from all study participants before enrolling them in the study.

\section{Conflicts of Interest}

The authors declare that there are no conflicts of interest.

\section{Acknowledgments}

The authors would like to express their gratitude to Dr. Devashree Warnekar and Dr. Pakadeesh Bharati for their immense help in writing the article; Dr. Parvathy Varma for laboratory support; and Mr. Shekhar E and Ms. Amisha S. for their help in data collection.

\section{References}

[1] E. Miserocchi, G. Fogliato, G. Modorati, F. Bandello, Review on the worldwide epidemiology of uveitis," European Journal of Ophthalmology, vol. 23, no. 5, pp. 705-717, 2013.

[2] M. D. de Smet, S. R. J. Taylor, B. Bodaghi, E. Miserocchi, P. I. Murray, and U. Pleyer, "Understanding uveitis: the impact of research on visual outcomes," Progress in Retinal and Eye Research, vol. 30, no. 6, pp. 452-470, 2011.

[3] T. Tsirouki, A. Dastiridou, C. Symeonidis, O. Tounakaki, I. Brazitikou, and C. Kalogeropoulos, "A focus on the epidemiology of uveitis," Ocular Immunology and Inflammation, vol. 26, no. 1, pp. 2-16, 2018.

[4] J. Biswas, S. Narain, D. Das, and S. K. Ganesh, "Pattern of uveitis in a referral uveitis clinic in India," International Ophthalmology, vol. 20, no. 4, pp. 223-228, 1996.

[5] J. H.-M. Chang and D. Wakefield, "Uveitis: a global perspective," Ocular Immunology and Inflammation, vol. 10, no. 4, pp. 263-279, 2002.

[6] M. R. Munk, M. Bolz, W. Huf, F. Sulzbacher, P. Roberts, and C. Simader, "Morphologic and functional evaluations during development, resolution, and relapse of uveitis-associated cystoid macular edema," Retina, vol. 33, no. 8, pp. 1673-1683, 2013.

[7] S. V. Branson, B. R. McClafferty, and S. K. Kurup, "Vitrectomy for epiretinal membranes and macular holes in uveitis patients," Journal of Ocular Pharmacology and Therapeutics, vol. 33, no. 4, pp. 298-303, 2017.

[8] E. D’Ambrosio, P. Tortorella, and L. Iannetti, "Management of uveitis-related choroidal neovascularization: from the pathogenesis to the therapy," Journal of Ophthalmology, vol. 2014, Article ID 450428, 6 pages, 2014.

[9] A. Heiligenhaus, K. Walscheid, and U. Pleyer, "Cataracts in uveitis," Klinische Monatsblaetter fuer Augenheilkunde, vol. 235, no. 5, pp. 568-575, 2018.

[10] A. D. Dick, N. Tundia, R. Sorg et al., "Risk of ocular complications in patients with noninfectious intermediate uveitis, posterior uveitis, or panuveitis," Ophthalmology, vol. 123, no. 3, pp. 655-662, 2016.

[11] M. Prete, S. Guerriero, R. Dammacco et al., "Autoimmune uveitis: a retrospective analysis of 104 patients from a tertiary reference center," Journal of Ophthalmic Inflammation and Infection, vol. 4, no. 1, p. 17, 2014.

[12] M. D. Becker and J. T. Rosenbaum, "Essential laboratory tests in uveitis," Uveitis Update, vol. 31, pp. 92-108, 1999.

[13] A. Rothova, H. J. Buitenhuis, C. Meenken et al., "Uveitis and systemic disease," British Journal of Ophthalmology, vol. 76, no. 3, pp. 137-141, 1992.

[14] D. A. Jabs, R. B. Nussenblatt, and J. T. Rosenbaum, "Standardization of uveitis nomenclature (SUN) working group. Standardization of uveitis nomenclature for reporting clinical data. Results of the first international workshop," American Journal of Ophthalmology, vol. 140, no. 3, pp. 509-516, 2005.

[15] A. Heiligenhaus, H. Michels, C. Schumacher et al., "Evidencebased, interdisciplinary guidelines for anti-inflammatory treatment of uveitis associated with juvenile idiopathic arthritis," Rheumatology International, vol. 32, no. 5, pp. 1121-1133, 2012.

[16] S. R. Rathinam, R. Krishnadas, R. Ramakrishnan et al., "Population-based prevalence of uveitis in Southern India," British Journal of Ophthalmology, vol. 95, no. 4, pp. 463-467, 2011.

[17] D. Das, H. Bhattacharjee, P. Bhattacharyya et al., "Pattern of uveitis in North East India: a tertiary eye care center study," Indian Journal of Ophthalmology, vol. 57, no. 2, pp. 144-146, 2009 Apr.

[18] J. Biswas, R. Kharel, and P. Multani, "Changing uveitis patterns in South India - comparison between two decades," Indian Journal of Ophthalmology, vol. 66, no. 4, pp. 524-527, 2018.

[19] T. Barisani-Asenbauer, S. M. Maca, L. Mejdoubi, W. Emminger, K. Machold, and H. Auer, "Uveitis- a rare disease often associated with systemic diseases and infectionsa systematic review of 2619 patients," Orphanet Journal of Rare Diseases, vol. 7, no. 1, p. 57, 2012.

[20] E. M. D’Ambrosio, M. La Cava, P. Tortorella, M. Gharbiya, M. Campanella, and L. Iannetti, "Clinical features and complications of the HLA-B27-associated acute anterior uveitis: a metanalysis," Seminars in Ophthalmology, vol. 32, no. 6, pp. 689-701, 2017.

[21] D. A. Brewerton, A. Nicholls, M. Caffrey, D. Walters, and D. C. O. James, "Acute anterior uveitis and HL-A 27," The Lancet, vol. 302, no. 7836, pp. 994-996, 1973.

[22] A. Rothova, M. S. Suttorp-van Schulten, W. Frits Treffers, and A. Kijlstra, "Causes and frequency of blindness in patients with intraocular inflammatory disease," British Journal of Ophthalmology, vol. 80, no. 4, pp. 332-336, 1996.

[23] O. M. Durrani, N. N. Tehrani, J. E. Marr, P. Moradi, P. Stavrou, and P. I. Murray, "Degree, duration, and causes of visual loss in uveitis," British Journal of Ophthalmology, vol. 88, no. 9, pp. 1159-1162, 2004.

[24] J. T. Rosenbaum, J. Ku, A. Ali, D. Choi, and E. B. Suhler, "Patients with retinal vasculitis rarely suffer from systemic 
vasculitis," Seminars in Arthritis and Rheumatism, vol. 41, no. 6, pp. 859-865, 2012.

[25] N. Cereb, C. Kim, A. L. Hughes, and S. Y. Yang, "Molecular analysis of HLA-B35 alleles and their relationship to HLA-B15 alleles," Tissue Antigens, vol. 49, no. 4, pp. 389-396, 1997.

[26] S. Itescu, U. Mathur-Wagh, M. L. Skovron et al., "HLA-B35 is associated with accelerated progression to AIDS," Journal of Acquired Immune Deficiency Syndromes, vol. 5, no. 1, pp. 37-45, 1992.

[27] R. Scorza Smeraldi, G. Fabio, A. Lazzarin et al., "HLA-associated susceptibility to AIDS: HLA B35 is a major risk factor for Italian HIV-infected intravenous drug addicts," Human Immunology, vol. 22, no. 2, pp. 73-79, 1988.

[28] R. E. Petty, D. W. C. Hunt, D. F. Rollins, M.-L. Schroeder, and M. L. Puterman, "Immunity to soluble retinal antigen in patients with uveitis accompanying juvenile rheumatoid arthritis," Arthritis \& Rheumatism, vol. 30, no. 3, pp. 287-293, 1987.

[29] J. J. Dubost, F. Demarquilly, M. Soubrier, C. Coussediere, J. M. Ristori, and B. J. Sauvezie, "HLA and self-limiting, unclassified rheumatism. A role for HLA-B35?" Journal of Rheumatology, vol. 26, no. 11, pp. 2400-2403, 1999.

[30] D. Šošo, J. Aljinović, I. Marinović, S. L. Kojundžić, E. Č Jeličić, and D. M. Krstulović, "The occurrence of sacroiliitis in HLA$\mathrm{B} * 35$-positive patients with undifferentiated spondyloarthritis. A cross sectional MRI study," Clinical Rheumatology, vol. 39, no. 8, pp. 2299-2306, 2020.

[31] N. S. W. Wilhelmsen, R. Weber, F. Monteiro, J. Kalil, and I. D. Miziara, "Correlation between histocompatibility antigens and recurrent aphthous stomatitis in the brazilian population," Brazilian Journal of Otorhinolaryngology, vol. 75, no. 3, pp. 426-431, 2009.

[32] Z. Ślebioda, E. Szponar, and A. Kowalska, "Recurrent aphthous stomatitis: genetic aspects of etiology," Advances in Dermatology and Allergology, vol. 30, no. 2, pp. 96-102, 2013.

[33] K. Karaman-Kraljević, V. Stambuk, N. Stambuk, and A. Kastelan, "A prospective study on the etiology of uveitis," Current Eye Research, vol. 9, no. Suppl, pp. 13-16, 1990.

[34] S. Lenna, S. Assassi, G. A. Farina et al., "The HLA-B*35 allele modulates ER stress, inflammation and proliferation in PBMCs from Limited Cutaneous Systemic Sclerosis patients," Arthritis Research and Therapy, vol. 17, no. 1, p. 363, 2015.

[35] K. McHugh and P. Bowness, "The link between HLA-B27 and SpA--new ideas on an old problem," Rheumatology, vol. 51, no. 9, pp. 1529-1539, 2012. 